\title{
DECLASSIFIED
}

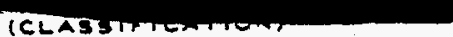

Undocumented

Eiv -43394

\section{GENERAL ELECTRL}

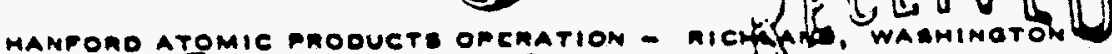

OATE

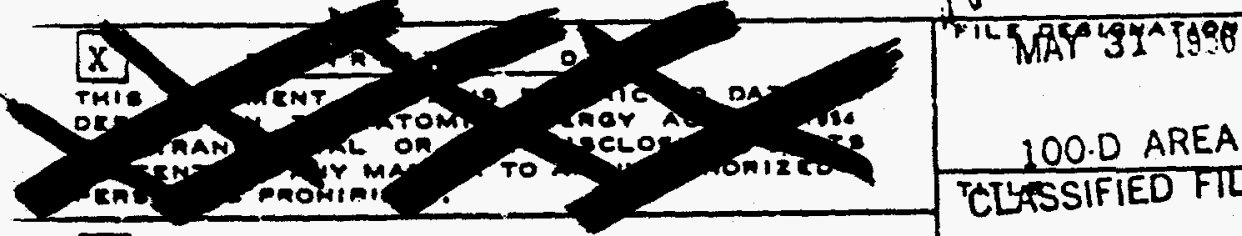

otmer of ricial classifico infonmation

TMIE MATERIAL CONTAINE INFORMATION AFFECTIMO

THE MATIOMAL DEPENEE OP TME UNITED ETATEE

WITHIN TME MEAMINO OP. THE cSPIONAGE LAWE,

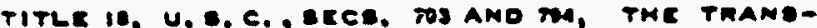

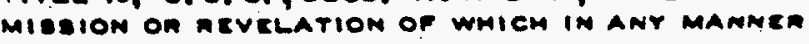
TO AN UNAUTMOnIzLO PEMLON IS PNOMIAITEO or

LAW.

\subsection{AREA TCLSSIFIED FILES}

TOW TEST - CG-4O2 "DR TTYPE" REAR PIOTAIL

WITH $B, D, D R$ \& I PILS FITTIMAS

\section{AUTHOA}

X. J. Bell

TMI8 DOCUMKMT MUST NOT OE LEFT UMATTEMOSO ON WHERE AN UMAUTHORIZEO PERBON MAY MAVK ACCESS THIB DOCUM

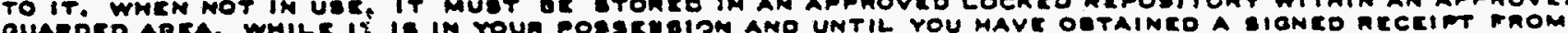

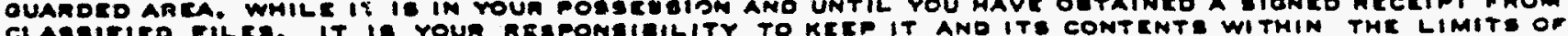
CLABEIFIED FILE, IT IS YOUR REBPOMEIEILITY TO KETP IT AND ITS CONTENTE WITHIN THE LIMITE OF THIE PROLECT AND FROM ANY UMAUTMONIZED PENSON, ITE TRANBMITTAL TO, AND STOAAOE AT YOUR PLACE

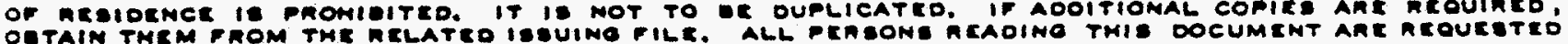
TO

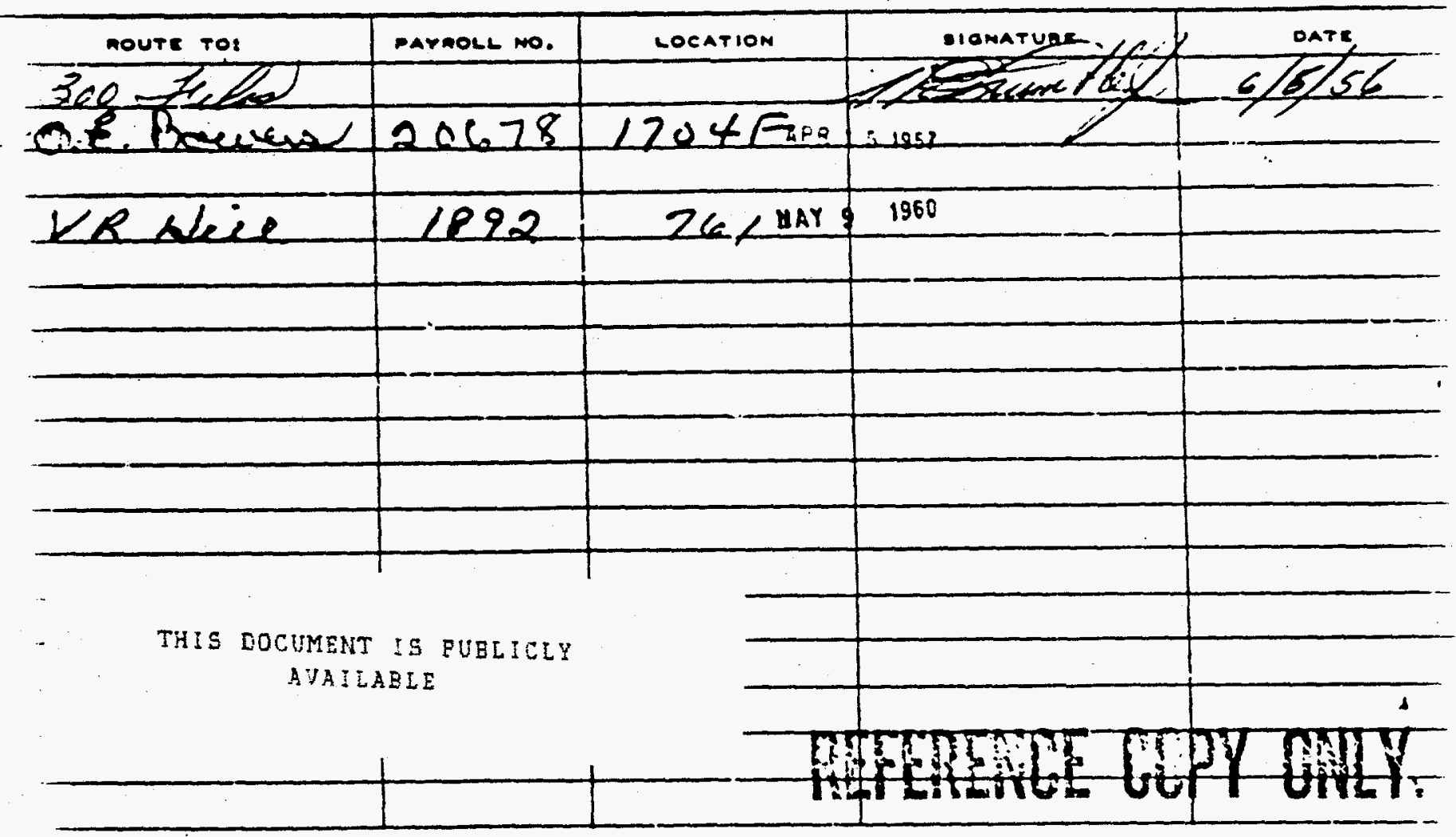

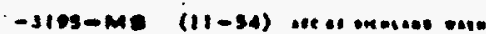




\section{DISCLAIMER}

This report was prepared as an account of work sponsored by an agency of the United States Government. Neither the United States Government nor any agency thereof, nor any of their employees, make any warranty, express or implied, or assumes any legal liability or responsibility for the accuracy, completeness, or usefulness of any information, apparatus, product, or process disclosed, or represents that its use would not infringe privately owned rights. Reference herein to any specific commercial product, process, or service by trade name, trademark, manufacturer, or otherwise does not necessarily constitute or imply its endorsement, recommendation, or favoring by the United States Government or any agency thereof. The views and opinions of authors expressed herein do not necessarily state or reflect those of the United States Government or any agency thereof. 


\section{DISCLAIMER}

Portions of this document may be illegible in electronic image products. Images are produced from the best available original document. 


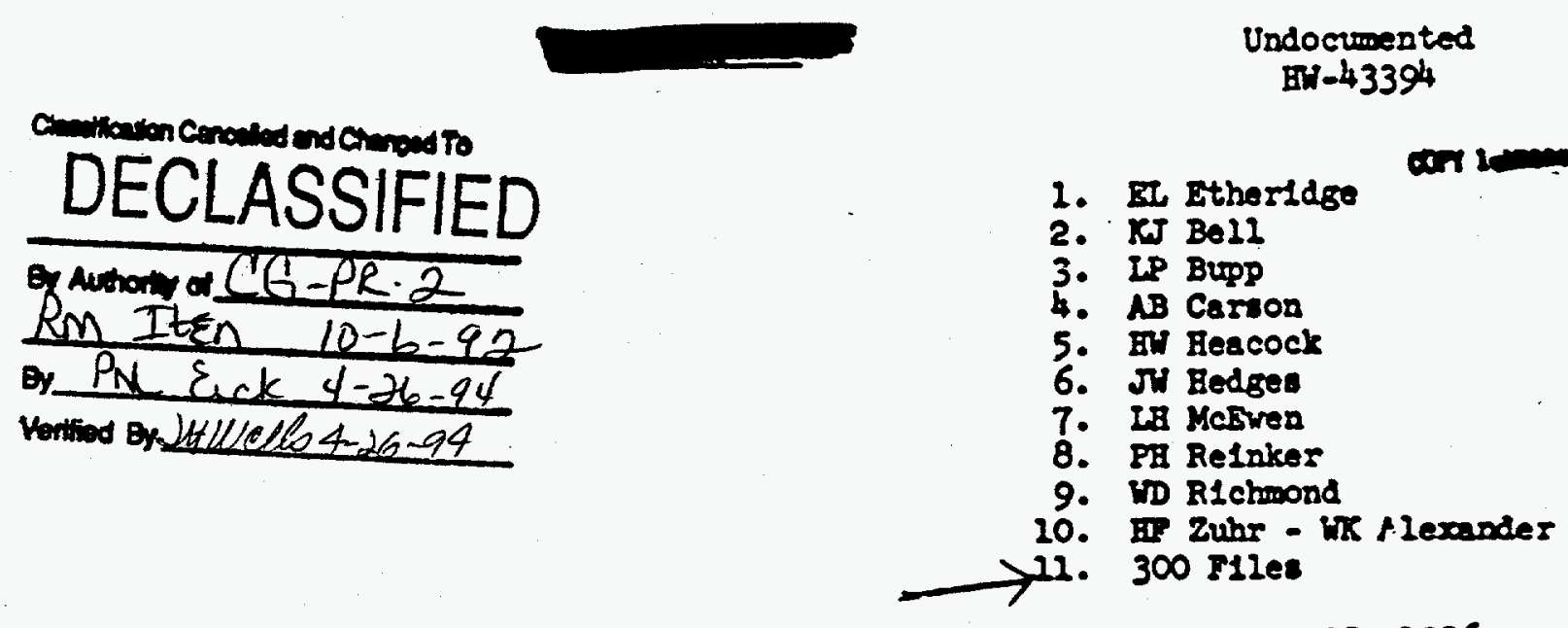

May 25, 1956

TO: B. L. Btber1d8e

FROK: K. J. Bell

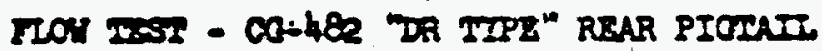
ITH B,D, DR \& PIIS FIMTIMGS

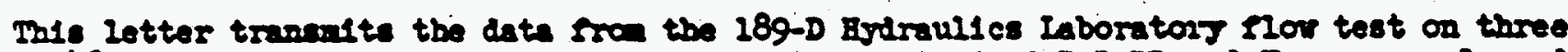

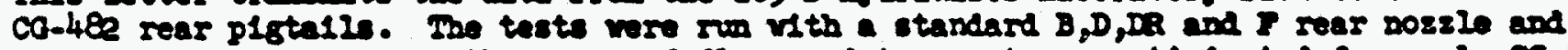

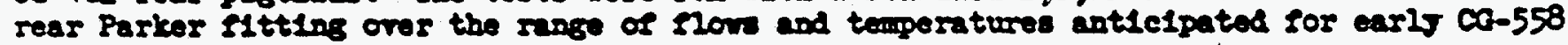
operation.

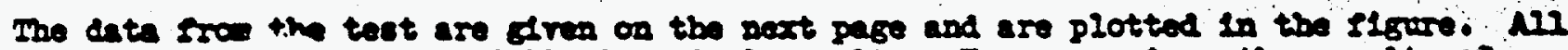

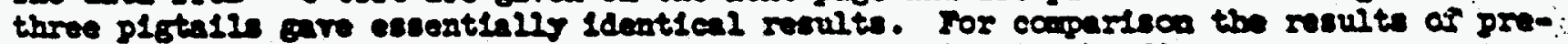
rlour teste rith present reer plotalle are also plotted in the rigure.

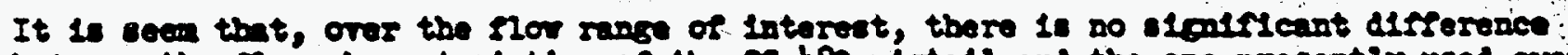

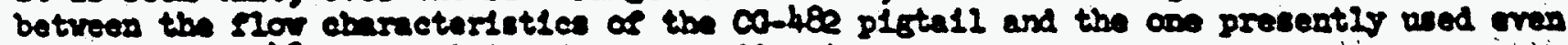

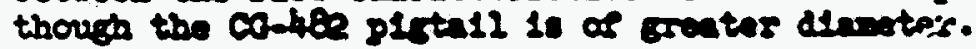

$\mathbf{m o p}$
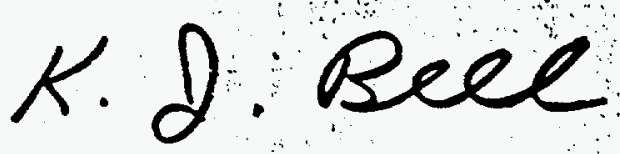

Approved by:
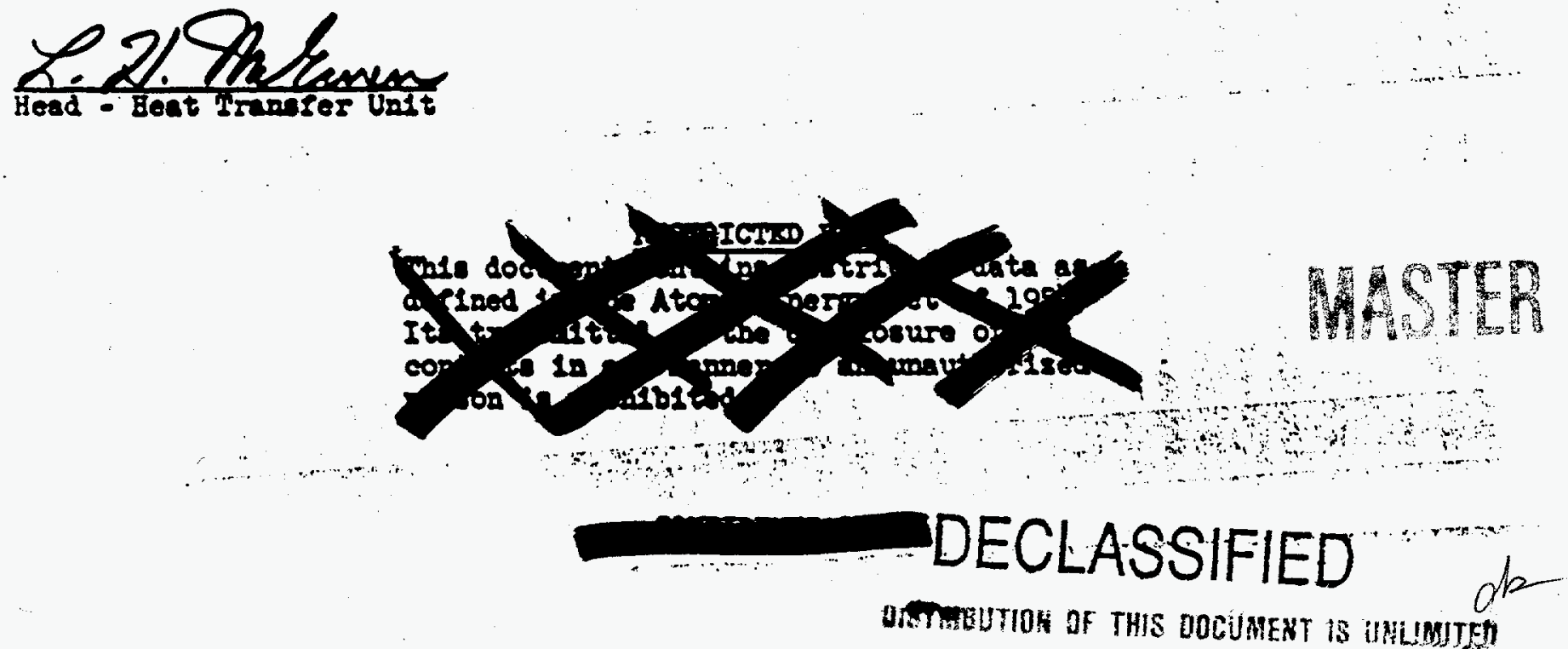

ORTMEUTIOK DF THIS DOCUMENT IS INLUMITSH 


\section{DECLASSIFIED}

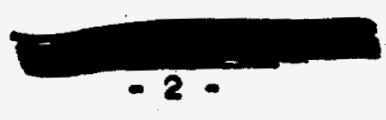

\section{Ondocruantod}

Bu-4339h

B,D,DR a IIIMIMCB, CO-48E

\section{P1gtall of}

\begin{tabular}{|c|c|c|c|c|c|c|c|c|c|}
\hline $\begin{array}{l}\text { Plor, Con } \\
\text { Rear Beader, pale } \\
\text { Rear Hossle Bnrrel, pele } \\
\text { Outlet Teup., "C }\end{array}$ & $\begin{array}{r}40.5 \\
30.5 \\
214 \\
22.0\end{array}$ & $\begin{array}{r}38.4 \\
30.0 \\
203.5 \\
12.0\end{array}$ & $\begin{array}{l}34.2 \\
30.0 \\
89 \\
11.5\end{array}$ & $\begin{array}{l}36.7 \\
30.5 \\
98.5 \\
100\end{array}$ & $\begin{array}{l}40.3 \\
30.5 \\
222.5 \\
100\end{array}$ & $\begin{array}{r}42.4 \\
32.0 \\
134.5 \\
100.5\end{array}$ & $\begin{array}{l}42.1 \\
29.5 \\
238.5 \\
120\end{array}$ & $\begin{array}{l}40.0 \\
30.0 \\
225.5 \\
212\end{array}$ & $\begin{array}{l}36.2 \\
28.5 \\
200 \\
109\end{array}$ \\
\hline \multicolumn{10}{|c|}{ P1stall of } \\
\hline 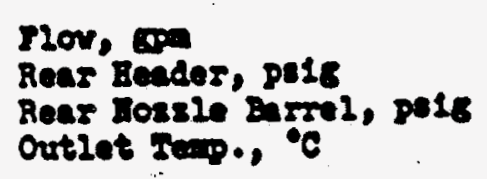 & $\begin{array}{r}40.4 \\
30.5 \\
214.5 \\
23.5\end{array}$ & $\begin{array}{l}38.25 \\
30.0 \\
203.5 \\
23.5\end{array}$ & $\begin{array}{l}34.25 \\
30.0 \\
88.5 \\
24.5\end{array}$ & $\begin{array}{l}36.2 \\
30.5 \\
99.5 \\
200\end{array}$ & $\begin{array}{l}40.15 \\
30.0 \\
122 \\
102\end{array}$ & $\begin{array}{l}42.15 \\
29.5 \\
135 \\
100\end{array}$ & $\begin{array}{l}42.1 \\
28.0 \\
135 \\
206\end{array}$ & $\begin{array}{l}40.0 \\
30.0 \\
123 \\
100\end{array}$ & $\begin{array}{l}35.9 \\
31.0 \\
202.5 \\
210\end{array}$ \\
\hline \multicolumn{10}{|c|}{ P1ota12 /3 } \\
\hline 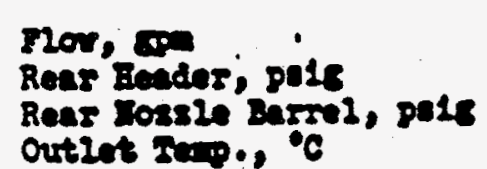 & $\begin{array}{r}10.3 \\
30.0 \\
213.5 \\
25.0\end{array}$ & $\begin{array}{c}38.2 \\
30.5 \\
103 \\
15.0\end{array}$ & $\begin{array}{l}34.2 \\
29.5 \\
08.5 \\
15.5\end{array}$ & $\begin{array}{l}36.1 \\
30.0 \\
99 \\
100\end{array}$ & $\begin{array}{c}40.0 \\
29.0 \\
223 \\
98.0\end{array}$ & $\begin{array}{l}42.1 \\
29.5 \\
136 \\
100.5\end{array}$ & $\begin{array}{r}42.7 \\
30.5 \\
232.5 \\
208.5\end{array}$ & $\begin{array}{r}39.6 \\
31.0 \\
121.5 \\
109\end{array}$ & $\begin{array}{l}35.5 \\
28.5 \\
102.5 \\
109\end{array}$ \\
\hline
\end{tabular}




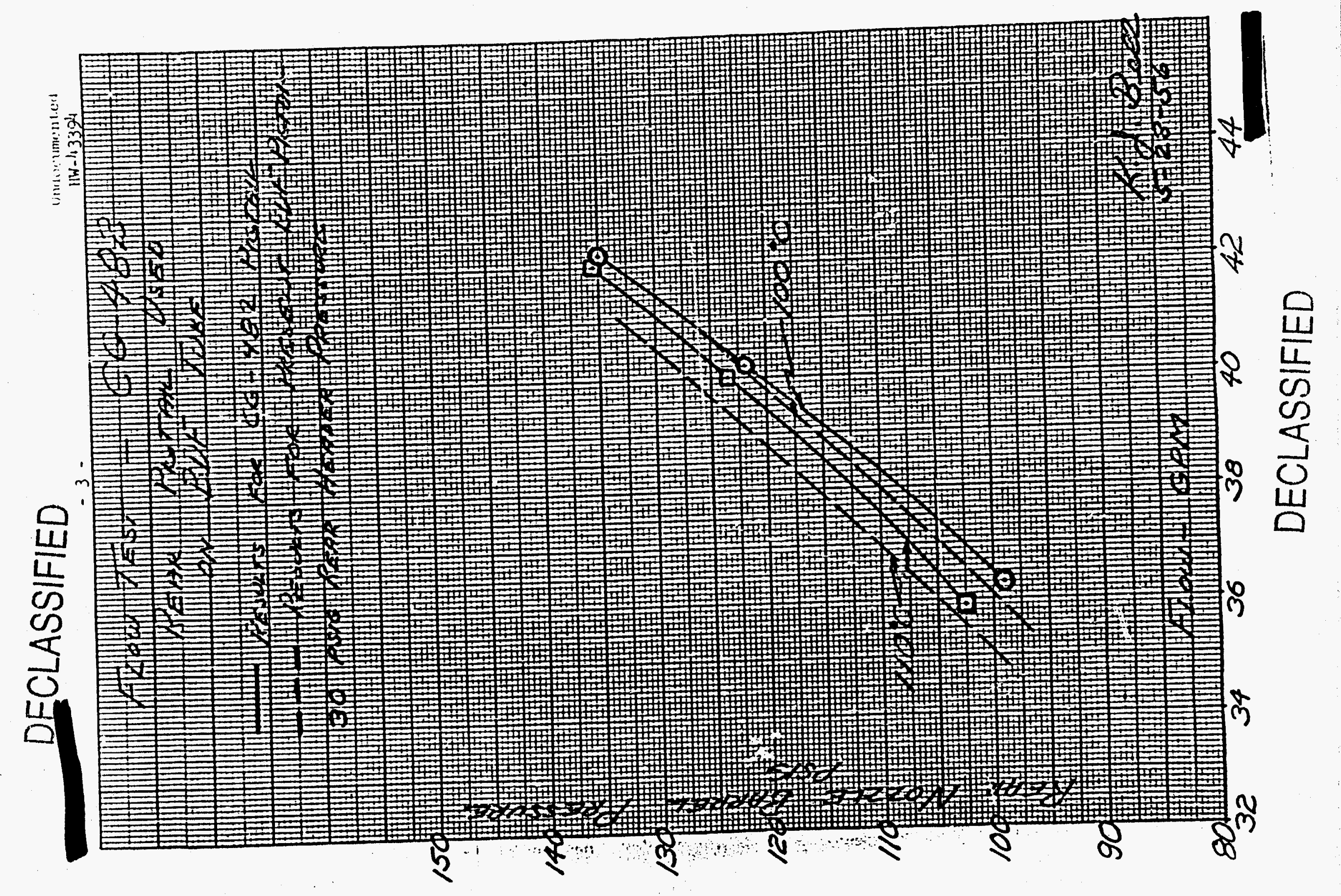

